\title{
Spontaneous Bacterial Infections in Cirrhosis- Peritonitis and Beyond
}

\author{
Deepinder Kaur ${ }^{1}$, Omesh Goyal ${ }^{2}$ \\ Department of Microbiology', Department of Gastroenterology ${ }^{2}$ \\ Dayanand Medical College and Hospital, Ludhiana, Punjab
}

Patients with liver cirrhosis have high morbidity and mortality due to either direct complications of the loss of liver function and/or portal hypertension such as jaundice, encephalopathy, variceal hemorrhage or indirect complications like hepatorenal syndrome and hepatocellular carcinoma. In addition, bacterial infections are another important cause of morbidity and mortality in cirrhotics. ${ }^{1}$ Predisposition of cirrhotics to various infections is mostly due to an associated immunocompromised state. ${ }^{2}$ Immune dysfunction in the cirrhotic patient is multifactorial. ${ }^{3}$ In cirrhotics, there is a decrease in bactericidal activity of phagocytic cells $;{ }^{1,4}$ and the complement levels, which are critical in bacterial phagocytosis, are also low. ${ }^{1,5}$ In addition, cirrhosis is accompanied by an impaired reticuloendothelial system (RES), the main defensive system against bacteremia and other infections acquired through a hematogenous route. Most of the RES activity is located in the liver where Kupffer's cells (tissue macrophages) are the major components. In cirrhosis, RES activity is impaired because of portosystemic shunting that bypasses the liver (thereby escaping the action of the RES) and because of impaired Kupffer's cell phagocytic activity. Patients with a decreased RES activity develop spontaneous bacteremia and spontaneous bacterial peritonitis (SBP) at a higher rate than patients with a normal RES. ${ }^{6}$ Other immune defects include reduced chemo attractant activity, decreased polymorphonuclear leukocyte activity and impaired bactericidal function of $\operatorname{IgM}$ for certain strains of Escherichia coli. ${ }^{1}$

The pathophysiological mechanism leading to the development of spontaneous bacterial infections in cirrhotics has been well described. ${ }^{1}$ Bacterial translocation (BT) is an important step in the development of infections in cirrhotics. BT refers to the migration of bacteria or bacterial products (lipopolysaccharides, endotoxins) from the lumen of the intestine to extraintestinal sites such as the mesenteric lymph nodes (MLN). Although passage of commensal bacteria to MLN is a normal process that results in bacterial destruction, BT can become clinically significant with a failure of local/systemic immune defense mechanisms as occurs in cirrhosis. ${ }^{7}$ Cirrhotics have increased bacterial translocation from gut due to altered intestinal immunity and bacterial overgrowth. ${ }^{1.8}$ This produces gram-negative bacteremia giving an opportunity for distant invasion by these micro-organisms. ${ }^{1}$ Once an overt infection occurs, it may lead to systemic inflammatory response syndrome/sepsis which can precipitate hypotension (severe sepsis), renal dysfunction, encephalopathy, and coagulopathy, that is, multiorgan failure. With further deterioration in the hemodynamic status, septic shock and death will ensue.

Various risk factors for the development of bacterial infections in a cirrhotic have been identified. However, the only sustainable reproduced risk factor is Child-Pugh stage. ${ }^{2,8-11}$ The relationship between variceal bleeding and infection risk has been assessed adequately in the existing literature. ${ }^{12,13}$ Other possible risk factors reported are alcohol consumption, low albumin levels, increased white blood cell count and previous history of infection. ${ }^{3,13}$

In prospective series, bacterial infections have been reported to occur in 32 to $34 \%$ of hospitalized patients with cirrhosis and in about $45 \%$ of those admitted with gastrointestinal hemorrhage. ${ }^{1,14}$ The most common of these infections and those considered characteristic of the disease are the so-called "spontaneous" infections, namely spontaneous bacterial peritonitis (SBP) (25-31\%), spontaneous bacterial empyema (SBE) ( 15\%), and spontaneous bacteremia $(\sim 12 \%)$ which share pathogenic mechanism and management. ${ }^{1}$ They are called spontaneous because there is no obvious source of bacteria that would explain their spread to ascites, pleural fluid, or blood, respectively.' However, cirrhotic patients are at risk for other common and uncommon bacterial infections also due to their 
immunocompromised state. These include urinary tract infection, skin and soft tissue infections (SSTI), meningitis, endocardititis, phlegmonous colitis, and hepatic abscess. Out of all these infections, SBP has been the most extensively studied infection. Various aspects of the pathophysiology, treatment and chemoprophylaxis of SBP have been adequately outlined. ${ }^{1}$ However, the impact of other bacterial infections in cirrhotics remains vaguely described. In a comprehensive review on infections in cirrhotics, Christou et al have described in detail the existing data on all types of bacterial infection in cirrhosis. ${ }^{3}$ It was noted that the relative frequency of individual infectious complication greatly varies. In a recent study from our institute, ${ }^{11} 1395$ patients with cirrhosis were prospectively studied for the presence of bacterial infections. Out of these, $19.4 \%$ were diagnosed to have bacterial infection. The frequency of various bacterial infections was- SBP-45\%, SSTI-32.8\%, UTI-24.5\%, bacteremia-12.9\%, respiratory tract infections- $8.9 \%$, and meningitis- $0.74 \%$. Twenty three percent patients had multiple infections. Strikingly, SSTIs were found to be the second most common infection, next only to SBP.

A causative micro organism is isolated in roughly half of the cases with an equal distribution between gram negative bacilli (GNB) and gram-positive cocci (GPC); however, GNB, mainly Escherichia coli, predominate in SBP, urinary tract infections and SSTI, while GPC predominate in pneumonia (mainly Streptococcus pneumoniae) and staphylococci in bacteremia associated with invasive procedures. ${ }^{1,11,15,16}$ In this issue of the journal, Thakur \& Chawla et al report a similar microbiological profile from the ascitic fluid analysis in cirrhotics.

The mortality rates after bacterial infection in cirrhotics vary in published studies, according to populations, type of infection, and etiology, so that the overall prognosis has remained poorly defined. ${ }^{15}$ With early diagnosis and proper antibiotic treatment, the mortality of SBP has decreased significantly over the years. Prophylaxis of infection is currently based on selective intestinal decontamination using orally administered, moderately absorbed quinolones such as norfloxacin. It is indicated in cirrhotic patients admitted with GI hemorrhage (short-term) and in the prevention of recurrent SBP (long-term). Primary prophylaxis of bacterial infections cannot be widely recommended until patients at higher risk are identified and/or non-antibiotic prophylactic measures are developed.

\section{REFERENCES}

1. Thulstrup AM, Sorensen HT, Schonheyder HC, Moller JK, Tage-Jensen U. Population-based study of the risk and short-term prognosis for bacteremia in patients with liver cirrhosis. Clin Infect Dis.2000;31:1357-61.

2. Tandon P, Garcia-Tsao G. Bacterial infections, sepsis and multiorgan failure in cirrhosis. Semin Liver Dis. 2008;28:26-42.

3. Christou L, Georgios PG, Falagas MF. Bacterial infection related morbidity and mortality in cirrhosis. Am J Gastroenterol. 2007;102:1510-7.

4. Garcia-Gonzalez M, Boixeda D, Herrero D. Effect of granulocyte-macrophage colony-stimulating factor on leukocyte function in cirrhosis. Gastroenterology 1993;105:527-31.

5. Finlayson ND, Krohn K, Fauconnet MH, Anderson KE. Significance of serum complement levels in chronic liver disease.Gastroenterology 1972;63:653-9.

6. Rimola A, Soto R, Bory F, Arroyo V, Piera C, Rodes J. Reticuloendothelial system phagocytic activity in cirrhosis and its relation to bacterial infections and prognosis. Hepatology 1984;4:53-8.

7. Garcia-Tsao G, Lee FY, Barden GE, Cartun R, West AB. Bacterial translocation to mesenteric lymph nodes is increased in cirrhotic rats with ascites. Gastroenterology 1995;108:1835-41.

8. Caly WR, Strauss E. A prospective study of bacterial infections in patients with cirrhosis. J Hepatol. 1993;18:353-8.

9. Runyon BA. Bacterial infections in patients with cirrhosis. J Hepatol. 1993;18:271-2.

10. Wyke RJ. Problems of bacterial infection in patients with liver disease. Gut. 1987;28:623-41.

11. Sood A, Midha V, Goyal O, Goyal P, Sood P, Sharma SK, Sood N. Skin and soft tissue infections in cirrhotics: a prospective analysis of clinical presentation and factors affecting outcome. Indian J Gastroenterol. 2014;33(3):281-4. 
12. Thalheimer U, Triantos CK, Samonakis DN, Patch D, Burroughs AK. Infection, coagulation, and variceal bleeding in cirrhosis. Gut. 2005;54:556-63.

13. Deschenes M, Villeneuve JP. Risk factors for the development of bacterial infections in hospitalized patients with cirrhosis. Am J Gastroenterol.1999;94:2193-7.

14. Borzio M, Salerno F, Piantoni L, Cazzaniga M, Angeli P, Bissoli F et al. Bacterial infection in patients with advanced cirrhosis: a multicentre prospective study. Dig Liver Dis 2001;33:41-8.

15. Guadalupe Garcia-Tsao. Bacterial infections in cirrhosis: treatment and prophylaxis. J Hepatol;42:S85-S92

16. Fernandez J, Navasa M, Gomez J, Colmenero J, Vila J, Arroyo V, et al. Bacterial infections in cirrhosis: epidemiological changes with invasive procedures and norfloxacin prophylaxis. Hepatology 2002;35:140-8. 